\title{
Search for PeVatrons at the Galactic Center using a radio air-shower array at the South Pole
}

\author{
A. Balagopal V. ${ }^{1, \mathrm{a}}$, A. Haungs ${ }^{2, \mathrm{~b}}$ (D) , T. Huege ${ }^{2, \mathrm{c}}$, F. G. Schröder ${ }^{1, \mathrm{~d}}$ \\ ${ }^{1}$ Institut für Experimentelle Teilchenphysik, Karlsruhe Institute of Technology (KIT), Karlsruhe, Germany \\ ${ }^{2}$ Institut für Kernphysik, Karlsruhe Institute of Technology(KIT), Karlsruhe, Germany
}

Received: 2 November 2017 / Accepted: 10 January 2018 / Published online: 6 February 2018

(C) The Author(s) 2018. This article is an open access publication

\begin{abstract}
The South Pole, which hosts the IceCube Neutrino Observatory, has a complete and around-the-clock exposure to the Galactic Center. Hence, it is an ideal location to search for gamma rays of $\mathrm{PeV}$ energy coming from the Galactic Center. However, it is hard to detect air showers initiated by these gamma rays using cosmic-ray particle detectors due to the low elevation of the Galactic Center. The use of antennas to measure the radio footprint of these air showers will help in this case, and would allow for a $24 / 7$ operation time. So far, only air showers with energies well above $10^{16} \mathrm{eV}$ have been detected with the radio technique. Thus, the energy threshold has to be lowered for the detection of gamma-ray showers of $\mathrm{PeV}$ energy. This can be achieved by optimizing the frequency band in order to obtain a higher level of signal-to-noise ratio. With such an approach, $\mathrm{PeV}$ gamma-ray showers with high inclination can be measured at the South Pole.
\end{abstract}

\section{Introduction}

The study of air showers using radio detection techniques, to date, has been mainly applied in the case of charged cosmicray measurements and neutrino searches [1,2]. Such showers have been detected with energy thresholds of at least a few tens of PeV. We show that this technique can also be used for $\mathrm{PeV}$ gamma ray astronomy, by lowering the energy threshold. This can be done by extending the frequency band of measurement to higher frequencies than those used by current radio air-shower arrays.

The Galactic Center has been identified as a source of gamma rays of $\mathrm{TeV}$ energy by H.E.S.S. [3]. The source of

\footnotetext{
a e-mail: aswathi.balagopal@kit.edu

be-mail: andreas.haungs@kit.edu

c e-mail: tim.huege@kit.edu

de-mail: frank.schroeder@kit.edu
}

this excess of $\mathrm{TeV}$ gamma rays has been traced to a PeVatron near the Galactic Center, in particular, close to the black hole Sgr A*. The H.E.S.S. data prefer a power law spectrum of $\mathrm{E}^{-2.3}$ with no cut-off. The spectrum of $\mathrm{TeV}$ gamma rays can be extrapolated to PeV energies. Detection of $\mathrm{PeV}$ gamma rays approaching from the Galactic Center, would strengthen the evidence of the existence of such a PeVatron.

Current efforts to look for PeVatrons from the Galactic plane with the IceCube Observatory involve the measurement of the neutrino and muon fluxes from possible sources in the northern sky and the southern sky, respectively $[4,5]$. Such searches then aim at explaining the contribution of PeVatrons to the knee of the cosmic-ray spectrum. Observing such PeVatrons using down-going muons will restrict the visible sky to that within the nearly vertical zenith angle range, due to limitations in the detector volume. Hence, Galactic Center observations with the help of down-going muons would be restricted.

Gamma rays of PeV energy, upon entering the Earth's atmosphere will produce air showers, similar to those produced by cosmic rays. These air showers can be detected on the ground using particle detectors and radio antennas. The Galactic Center is always visible at the South Pole, at an angle of $29^{\circ}$ above the horizon (zenith angle of $61^{\circ}$ ). Hence the IceCube Observatory at the South Pole is an ideal location to search for gamma rays from the Galactic Center. The number of gamma rays arriving at the IceCube Observatory from the Galactic Center with energies above $0.8 \mathrm{PeV}$ is estimated to be around 11.5 events per year, from a simple extrapolation of the spectrum measured by H.E.S.S. (see Appendix A).

The IceCube Neutrino Observatory [6], the $1 \mathrm{~km}^{3}$ array for the detection of astrophysical neutrinos, has a surface component of ice-Cherenkov particle detectors (IceTop) used for the detection of cosmic-rays [7]. It is planned to upgrade IceTop using scintillators [8]. It is also foreseen to have a large surface array of scintillation detectors and air-Cherenkov telescopes as a part of IceCube-Gen2 [9-12]. A surface array of 
radio antennas in addition to this could potentially increase the accuracy for the detection of air showers (especially inclined air showers) and for the determination of mass composition. RASTA, a previous study that was made using test antennas at the South Pole explored the possibility of improving veto capabilities and cosmic-ray studies at IceCube [13]. Apart from this, in-ice radio measurements at the South Pole is also being made using ARA, that aims at measuring the radio signals produced by high energy neutrinos inside ice [14].

A surface array of radio antennas at the South Pole can be used to search for air showers produced by PeV gamma rays arriving from the Galactic Center. Inclined air showers of PeV energy will be hard to detect and reconstruct effectively using particle detectors, since a major part of the shower dies out by the time it reaches the detector array. This is especially the case for showers induced by gamma rays. Gamma-ray showers have lesser muonic content when compared to hadronic showers. In particular, the showers induced by protons have a significantly larger fraction of muons than gamma-ray showers. Muons, unlike electrons and positrons, are the most prominent component of inclined air showers of PeV energy, that will reach the ground. The low muonic content of gamma-ray showers results in fewer pulses in the IceTop tanks and the future scintillation detectors. In contrast, the radio signal from a shower with the same primary energy survives and can be detected on the ground by an array of radio antennas. Thus by comparing the radio emission to the number of muons detected on the ground one can distinguish between showers initiated by gamma rays and those by other nuclei.

Radio emission of air showers develops mainly due to the deflection of the electrons and positrons of the shower in the Earth's magnetic field (Geomagnetic effect). This

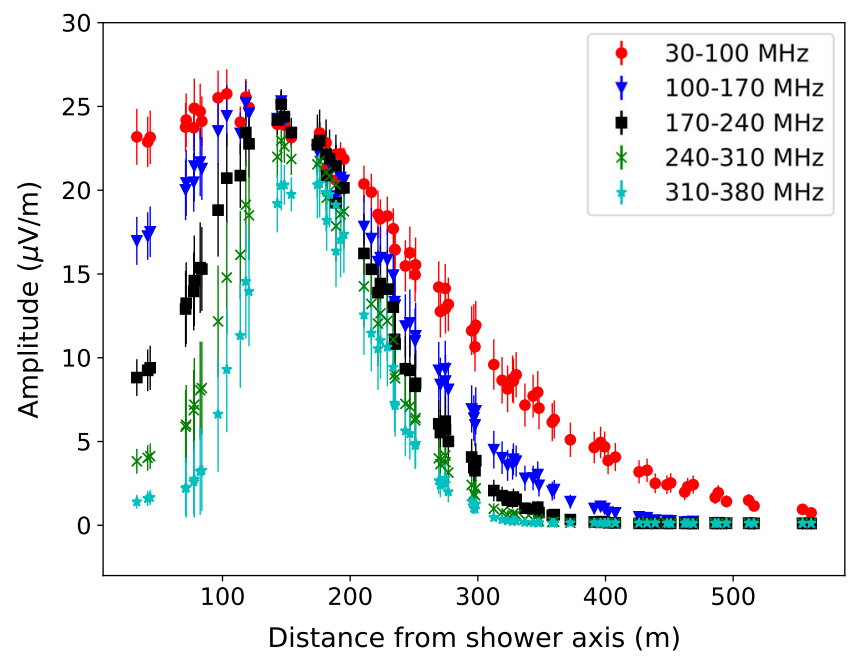

(a) $10 \mathrm{PeV}$ gamma-ray showers, $\theta=61^{\circ}, \alpha=79^{\circ}$ results in a time-varying current that produces radio pulses $[15,16]$. Another contribution to radio emission comes from the Askaryan effect, which is due to the charge excess at the shower front that forms as the shower propagates through the atmosphere $[17,18]$. This effect, which has a smaller influence in air showers than the geomagnetic effect, causes a small asymmetry in the total radio emission. At higher frequencies, a Cherenkov ring is visible in the radio footprint, due to the time compression of radio pulses caused by the refractive index of air [19]. For example, at frequencies such as that covered by the band $50-350 \mathrm{MHz}$, which is used by the lower frequency component of the Square Kilometer Array (SKA-LOW) [20], the Cherenkov ring is visible. Such a Cherenkov ring is only marginal in the frequency band $30-80 \mathrm{MHz}$, which is the frequency range used by most of the existing radio air shower experiments, e.g. AERA [21], Tunka-Rex [22] and LOFAR [23].

An inclined shower produced by a gamma ray from the Galactic Center will leave a large radio footprint on the ground, whose diameter ranges from several $100 \mathrm{~m}$ to $\mathrm{km}$ depending on the angle of inclination. Recent studies of inclined air showers by the Auger Engineering Radio Array (AERA) have experimentally proven this [24]. The footprint detected on the ground is elliptical in shape because of pure geometrical reasons [25]. The inclined air showers detected by AERA have energies higher than $10^{18} \mathrm{eV}$. Similar characteristics will be seen by showers of $\mathrm{PeV}$ energy arriving at the IceCube location.

Figure 1 shows the different simulated amplitudes delivered to 81 antennas, on an area of $1 \mathrm{~km}^{2}$, at the location of the IceTop stations [7]; that is with one antenna placed at the center of the two Cherenkov tanks that form an IceTop station (see also Fig. 5). The lateral distribution of the amplitudes in the figure are those from gamma-ray induced showers and

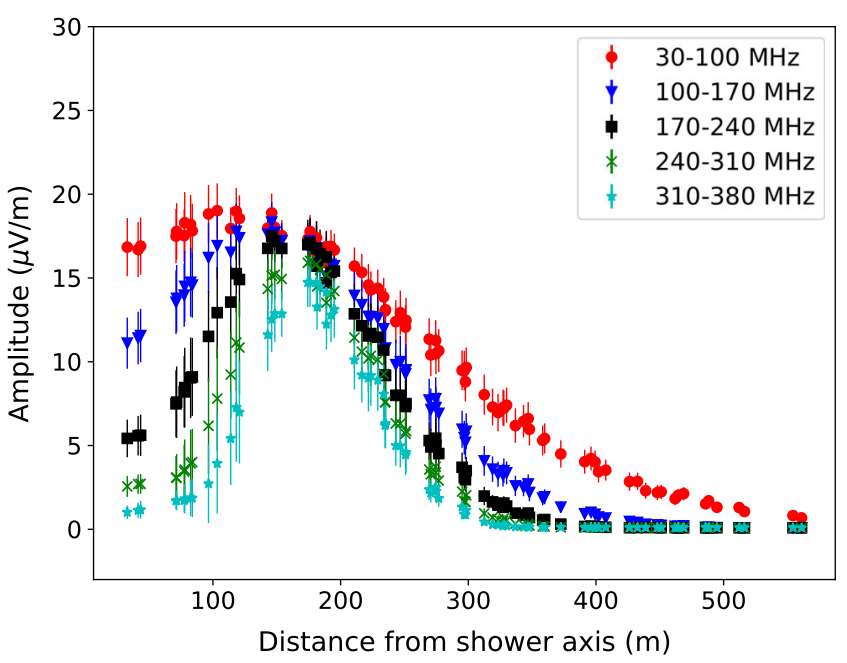

(b) $10 \mathrm{PeV}$ proton showers, $\theta=61^{\circ}, \alpha=79^{\circ}$

Fig. 1 Lateral distribution of radio signals for gamma-ray and proton showers for frequencies from 30 to $380 \mathrm{MHz}$. For illustration the signals are shown in various frequency bands with a width of $70 \mathrm{MHz}$ each 
proton induced showers at these antenna locations. Here, the zenith angle is fixed to $61^{\circ}$. These showers have a geomagnetic angle (angle between the Earth's magnetic field and the shower axis) of $\alpha=79^{\circ}$. For illustration, the frequencies are split into bands with a width of $70 \mathrm{MHz}$ each, and range from $30 \mathrm{MHz}$ all the way up to $380 \mathrm{MHz}$. The proton showers have lower amplitudes than gamma-ray showers since they have lower electromagnetic content. The plot shows the mean amplitude along with the spread about the mean with 30 simulated showers for gamma-ray and proton primaries. We can see that the lateral distribution for these showers change with the frequency of observation. At frequencies above 100 $\mathrm{MHz}$, we start to see the Cherenkov ring in such a distribution. At very high frequencies like those above $300 \mathrm{MHz}$, the emission becomes extremely localized, giving non-zero values of the amplitude only on the Cherenkov ring. That is, the radio signal dies out gradually at every other location at such high frequencies.

So far, it has been considered that air showers from cosmic rays with an energy range greater than $10^{16} \mathrm{eV}$ can be measured using the radio detection technique. At energies lower than this, the background overwhelms the radio signal from an air shower [1]. This is especially the case for the frequency range of 30-80 MHz. This makes it hard to measure air showers at low energies, unless interferometric methods are used. Thus in order to measure air showers produced by $\mathrm{PeV}$ gamma rays, we explore a different method to lower the energy threshold. This paper focuses on this aspect, especially on the optimization of the observing frequency bands in order to lower the threshold energy for the detection of gamma-ray air showers.

\section{Simulation of air showers}

A thorough study of the air showers that are produced by the incoming gamma rays is needed for predicting the radio signal that will be detected on the ground. For this purpose, air shower simulations were performed using CoREAS [26], which is the radio extension of CORSIKA [27]. We use CORSIKA-7.4005 with hadronic interaction models FLUKA-2011.2c.2 and SIBYLL-2.1 [28]. Later simulations used CORSIKA-7.5700 with SIBYLL-2.3. This was not seen to change the received radio signals from air showers significantly (since the main difference between the versions is in the muonic content of the hadronic interactions). A total of 1579 simulations have been done for this study. The simulations used the atmosphere of the South Pole (South pole atmosphere for Oct. 01, 1997 provided in CORSIKA) with an observation level of $2838 \mathrm{~m}$ above the sea level. All the showers have been simulated using the thinning option (with a thinning level of $2.7 \times 10^{-7}$ ).
The showers simulated are those of gamma-ray primaries with energies ranging from 1 to $10 \mathrm{PeV}$. The azimuth angle for preliminary studies were fixed so that the shower axis is oriented anti-parallel to the Magnetic North $(\phi=0)$, thereby giving a geomagnetic angle of $79^{\circ}$ for a shower with a zenith angle of $61^{\circ}$. At the South Pole, the magnetic field is inclined at an angle of $18^{\circ}$ with respect to the vertical, with an intensity of $55.2 \mu \mathrm{T}$. The zenith angle is fixed to $61^{\circ}$ for a major portion of the simulations since this is the inclination of the Galactic Center at the South Pole. The core position was set at the center of the IceTop array, i.e. at $(0,0)$. For comparison, proton showers were also simulated, with the same parameters. The simulations included 81 antennas, each at the center of an IceTop station. This resulted in an array where the average antenna spacing is around $125 \mathrm{~m}$. An inner infill array of antennas with much denser spacing (approximately $90 \mathrm{~m}$ ) is also present, since such a structure is present for the IceTop stations also. The entire array covers an area of around $1 \mathrm{~km}^{2}$.

The output from CoREAS simulations gives the signal strength at each of these antenna stations in units of $\mu \mathrm{V} / \mathrm{m}$. This has to be folded through the response of an antenna, in order to estimate the measurable signal. For this purpose, a simple half wave dipole antenna with resonance at $150 \mathrm{MHz}$ was simulated using NEC2++ [29]. Antennas in the eastwest and in the north-south direction (with respect to the magnetic field) were used at the location of each station, to extract the complete signal from the air shower. Here, the zcomponent is neglected. This can be safely done because of the small angle between the magnetic field and the vertical, thereby resulting in a smaller $\mathrm{z}$ component of electric field as compared to the $\mathrm{x}$ and $\mathrm{y}$ components, even for the inclined air showers.

The simulations that are performed here are simplified, since the main focus is to understand the required experimental setup for lowering the energy threshold in order to detect PeV gamma rays from the Galactic Center. Specific effects like the impact of an optimized type of antenna or other details that can be important for a particular experimental setup are ignored in this context and can be included in the case of a more detailed study.

\section{Inclusion of a noise model}

One of the major challenges for the detection of radio signals from showers of $\mathrm{PeV}$ energy is the lower level of signal, when compared to the background noise as discussed in Sect. 1 . There can be external as well as internal (thermal) sources of noise for radio air shower experiments. The external sources of noise range from Galactic noise through man-made noise to noise contributed by atmospheric events. At the South Pole, the external contribution mainly comes from Galactic noise as the contributions from other elements are expected 
to be much lower in comparison. Existing air shower experiments point out that measurement of signals from inclined air showers of $\mathrm{PeV}$ energy range using the band of 30-80 $\mathrm{MHz}$ is hard to achieve. Within this frequency band, the signal will be dominated by noise, especially for showers in the $\mathrm{PeV}$ energy range.

In this study, a simplified and average model of diffuse Galactic noise developed by Cane [30] is used. It has already been shown by measurements from RASTA and ARA that the Cane model describes the Galactic noise measured at the South Pole with a reasonable accuracy $[31,32]$. The noise is given in units of brightness (Galactic Brightness background) in this model, as can be seen in Fig. 2. The corresponding brightness temperature, obtained from the relation $T=\frac{1}{2 k_{\mathrm{B}}} \frac{c^{2}}{v^{2}} B(v)$, is used for determining the Galactic contribution to the total noise. In addition to the Galactic noise, there is also a contribution from the thermal component, which arises due to the electronic boards and other equipments related to the experimental setup (internal noise). A thermal noise of $300 \mathrm{~K}$ is used here. With a very simple hardware, the thermal noise contribution could even be more than $300 \mathrm{~K}$. Much lower noise levels of a few $10 \mathrm{~K}$ can be achieved by dedicated hardware optimization.

The noise temperature can be related to the power received in the antenna by $P=k_{\mathrm{B}} T \delta v$, where $\delta v$ is the frequency interval within which the power is extracted and $k_{\mathrm{B}}$ is the Boltzmann constant. From Fig. 2, we see that the Galactic noise diminishes as the frequency increases. At frequencies above $\approx 150 \mathrm{MHz}$, we become mainly limited by the thermal noise.

The expected noise for a given frequency band can be expressed as time traces from the predicted noise temperature within this band (see Appendix B). Noise traces extracted like this can be compared to signals from air showers as shown in Fig. 3. Here, the signals considered are those for a gamma-ray shower of $10 \mathrm{PeV}$ energy and inclined at an angle of $61^{\circ}$.

It is clearly seen that the signal-to-noise ratio increases as we move to higher frequencies, as is expected from the behavior of the Galactic noise. Here, the signal-to-noise-ratio is determined as SNR $=S^{2} / N^{2}$ where $S$ is the maximum of the Hilbert envelope over the signal and $N$ is the rms noise in the specified frequency band. The suppression of the Galactic noise beyond $150 \mathrm{MHz}$ is visible in the time traces of the noise. It becomes obvious that moving on to higher frequencies will enable us to have a higher level of signalto-noise ratio (SNR), provided the antenna falls within the footprint of the shower.

\section{Optimizing the observing frequency band}

Although it is clear from Fig. 3 that using frequency bands that are higher than the standard band $(30-80 \mathrm{MHz})$ will

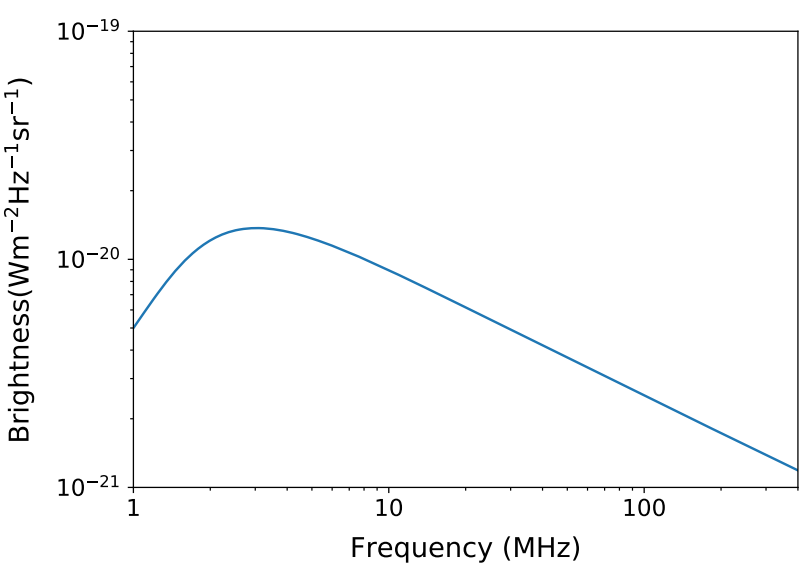

(a)

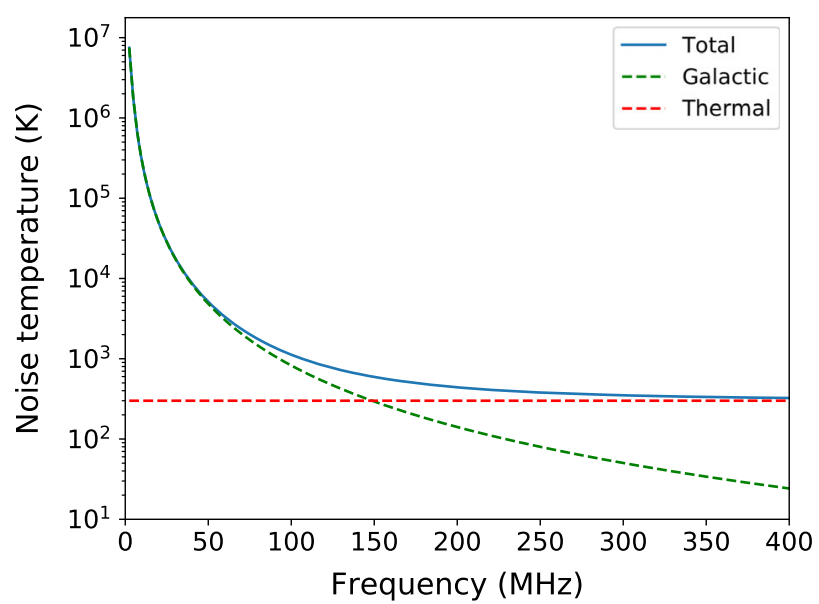

(b)

Fig. 2 a Brightness of the Galactic radio background radiation given by the Cane function [30]. $\mathbf{b}$ Total noise temperature as a function of frequency

help us in enhancing the signal-to-noise ratio, the exact band that should be used for maximizing the chances of observation is still unclear. It is of course, possible to measure in wide band frequencies, and then to digitally filter into the required frequency range. But this will increase the cost of the experiment considerably, since the usage of higher frequencies require a greater sampling rate and hence better communication facility, memory, ADC, etc. Thus, a detailed study is made to estimate the frequency range that will give a maximum signal-to-noise ratio (and thereby maximize the detection probability), and can hence be used for the experiment, which is the focus in the following section.

A close inspection of the shower footprint at higher frequencies reveals that there are three regions of interest: on the Cherenkov ring, inside the Cherenkov ring, and outside the Cherenkov ring. It is desirable to have a high value of SNR in all of these regions for maximizing the probability of detection in the entire antenna array. 


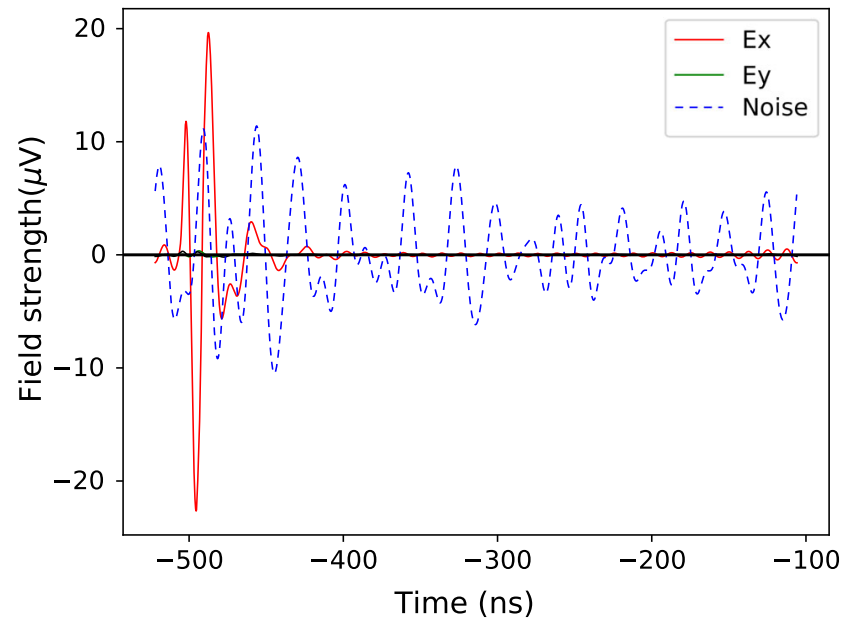

(a) $30-80 \mathrm{MHz},(\mathrm{SNR} \approx 35)$

Fig. 3 Simulated signal for a $10 \mathrm{PeV} \gamma$-ray shower and noise at a station located on the Cherenkov ring, with a distance of $107 \mathrm{~m}$ from the shower axis. The y-component of the signal shown here is nearly zero.

A scan of the possible frequency bands that can be used for the measurement of air showers of energy $10 \mathrm{PeV}$ is made. That is, we can construct a heat map of the SNR in different frequency bands. The frequencies for the heat map range from 30 to $150 \mathrm{MHz}$ for the lower edge of the frequency band and from 80 to $350 \mathrm{MHz}$ for the upper edge of the band. Such a scan is made for antenna stations at each region mentioned above. This is shown in Fig. 4 for a typical gamma-ray shower with a zenith angle of $61^{\circ}$ and an energy of $10 \mathrm{PeV}$.

It is obvious that the typical frequency band of $30-80 \mathrm{MHz}$ (lower left bins in Fig. 4) is not ideal for obtaining an optimal level of SNR. In the figure, the brightest zone for each region on the shower footprint shows the ideal frequency band, where a maximum range of SNR is obtained. Taking measurements at frequencies like 100-190 MHz gives a higher SNR. All bands where a value of SNR less than 10 is obtained are set to the color white, since this is the typical threshold for detection in an individual antenna station [33]. The bands with high SNR become especially crucial, when the energy threshold is attempted to be lowered. A map of the SNR that is measurable by the antennas is shown in Fig. 5. The black dots represent the 81 antennas considered in the simulations. The antennas considered for the frequency band scan in Fig. 4 are also marked here. The SNR map shown in the figure is obtained for the frequency band $100-190 \mathrm{MHz}{ }^{1}$

Showers of other zenith angles and other primaries also show a similar behavior in the frequency band scan. As the zenith angle and the primary type changes, there is a variation in the scaling of SNR. This results from the change in the total electromagnetic content (for different primary type) and the

1 This was produced by running a CoREAS simulation in parallel mode with 3750 antennas using the hadronic interaction model UrQMD instead of FLUKA.

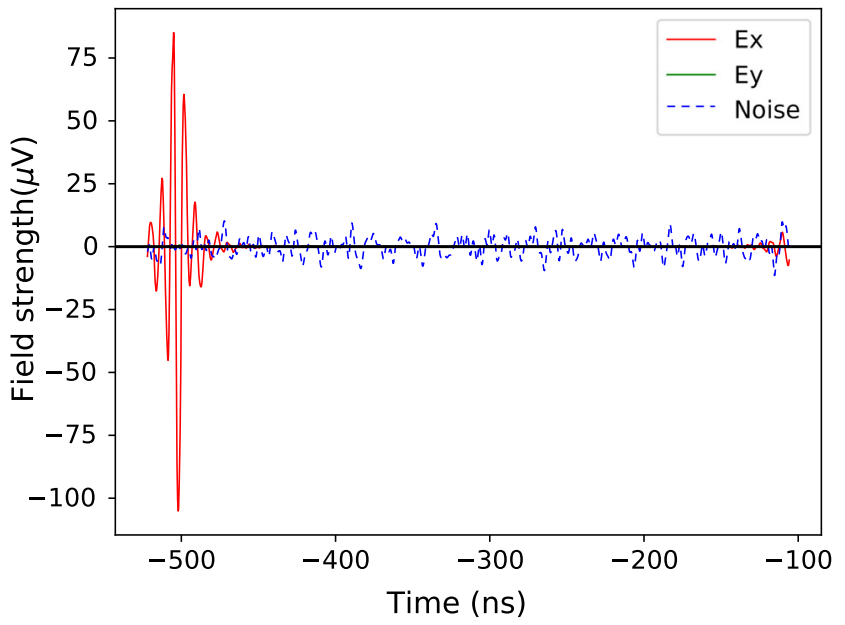

(b) $50-350 \mathrm{MHz},(\mathrm{SNR} \approx 1055)$

The received noise is much lower at higher frequencies, leading to a higher signal-to-noise ratio

different spread of the signal strength on the ground (for different zenith angle). There is a direct relation between the spread in the diameter of the Cherenkov ring and the inclination of the shower. Thus, the frequency bands with a higher value of SNR are the same for showers of other primaries and other zenith angles as that for a shower of zenith angle $61^{\circ}$ (shown in Fig. 4).

The observed signal-to-noise ratio in the antennas will depend on the energy of the shower, the zenith angle, and the azimuth angle (resulting in varying values of the Geomagnetic angle). The study of SNR in these parameter spaces is described in the following sections. The variation of the SNR with respect to the changing position of the shower maximum is not taken into account over here.

\subsection{Dependence on the zenith angle}

The evolution of the SNR with the zenith angle can be looked at for different frequency bands. This evolution is looked at for antenna stations at various perpendicular distances to the shower axis (which is equivalent to the radial distance of the antennas to the shower axis in the shower plane). Such an evolution is shown for zenith angles ranging from $0^{\circ}$ to $70^{\circ}$ in Fig. 6, for the bands 30-80, 100-190 and 50-350 MHz.

For the standard band of $30-80 \mathrm{MHz}$, the signal-to-noise ratio is significantly lower than that for the bands 50-350 and 100-190 MHz. Among all the three bands, the highest level of signal-to-noise ratio is obtained for $100-190 \mathrm{MHz}$ for all zenith angles, as expected. In particular, for showers of greater inclination, a higher signal-to-noise ratio is achieved in most of the antennas if we use the higher frequency bands. The areas where the Cherenkov ring falls on the antennas are visible for the higher frequencies. These are the really bright 


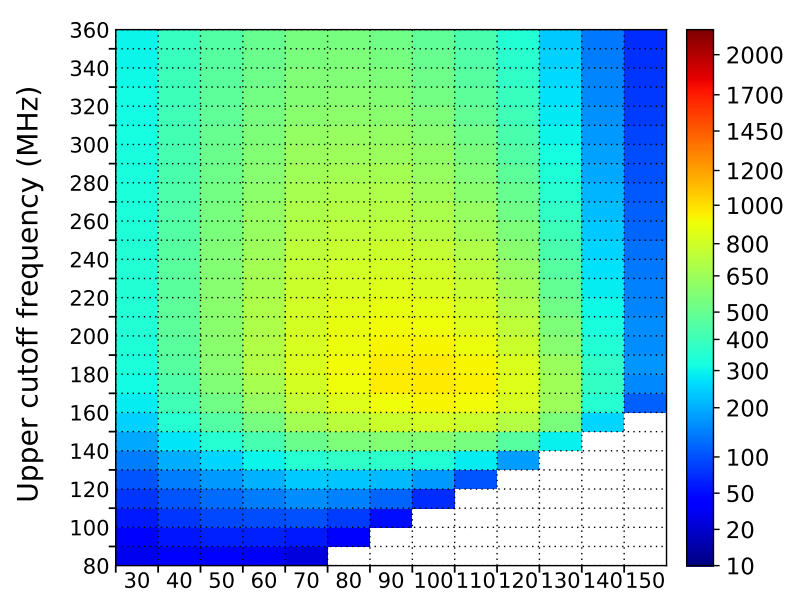

Lower cutoff frequency $(\mathrm{MHz})$

(a) Inside the Cherenkov ring (distance: $34 \mathrm{~m}$ )

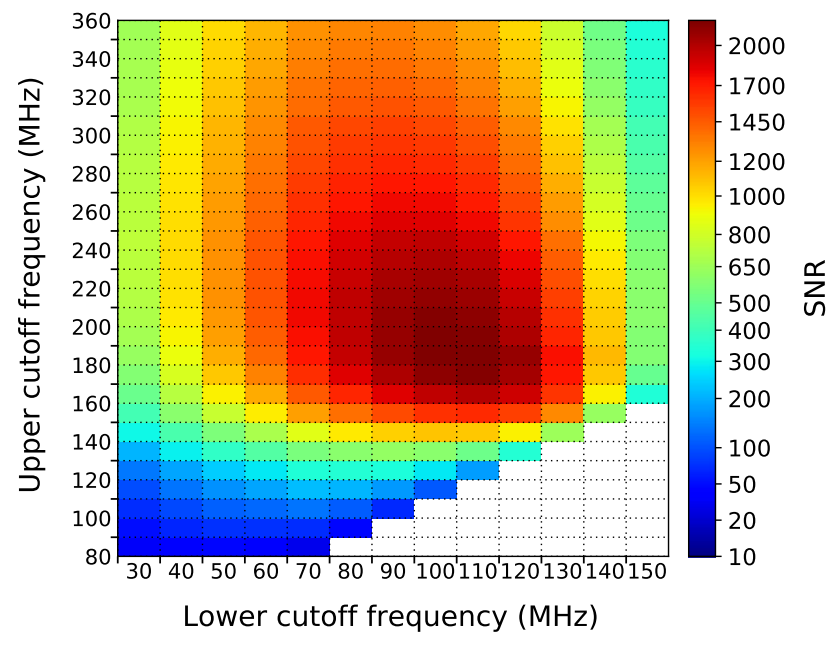

(b) On the Cherenkov ring (distance: $107 \mathrm{~m}$ )

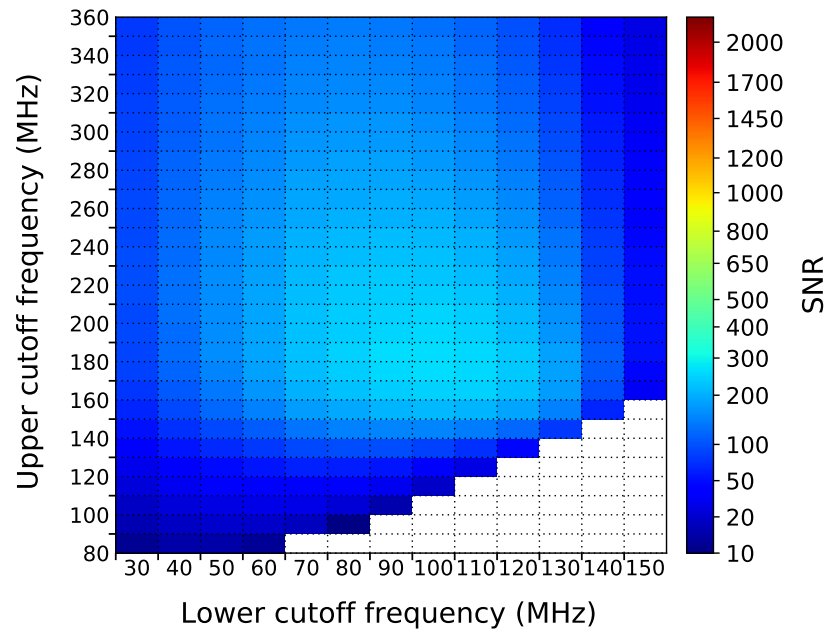

(c) Outside the Cherenkov ring (distance: $246 \mathrm{~m}$ )

Fig. 4 SNR seen in a typical antenna inside, on and outside the Cherenkov ring respectively, at various frequency bands, for one typical shower induced by a $10 \mathrm{PeV}$ gamma-ray primary with zenith angle $=61^{\circ}$ and $\alpha=79^{\circ}$

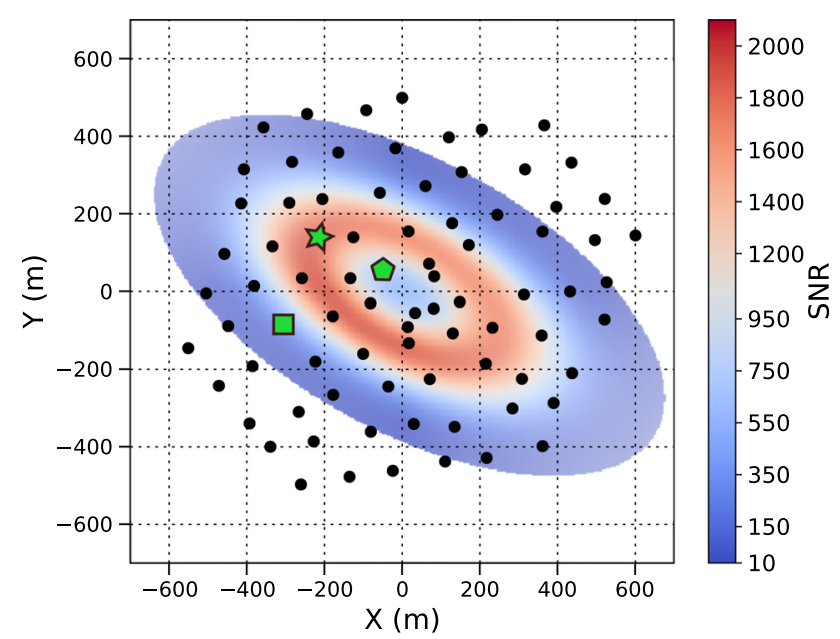

Fig. 5 SNR map of a $10 \mathrm{PeV}$ gamma-ray $\operatorname{shower}\left(\theta=61^{\circ}\right)$ at $100-190$ $\mathrm{MHz}$. The black dots represent the 81 antenna positions. The antenna on the Cherenkov ring used for the frequency band scan is shown by the star shape. The square shape represents the antenna outside the Cherenkov ring and the pentagon that inside the Cherenkov ring

regions seen for each zenith angle and appears only for the more inclined showers.

At lower zenith angles, a major part of the shower is lost because of clipping effects. The high observation level at the South Pole is the reason for the showers getting clipped off. The distance to the shower maximum at these zenith angles is about a few kilometers, while that for showers of $70^{\circ}$ inclination is in the order of tens of kilometers. The clipping of the shower at lower zenith angles causes the radio emission to be underdeveloped for detection. This is also the reason for the appearance of the Cherenkov ring only for zenith angles $\gtrsim 30^{\circ}$.

In Fig. 6, the distances of the antennas from the shower axis fall within the range of $50 \mathrm{~m}$ to approximately $520 \mathrm{~m}$, but only the antennas with a SNR $>10$ can detect these showers. For vertical showers, these are the antennas with distances of $\approx 100 \mathrm{~m}$ and for inclined showers, these are the antennas that are even as far away as $500 \mathrm{~m}$. This range corresponds to the required minimum spacing to detect these showers. That is, for vertical showers the antennas could at most have a spacing of $100 \mathrm{~m}$ and for inclined showers with $\theta \gtrsim 60^{\circ}$ a spacing of $300 \mathrm{~m}$ is sufficient to achieve a threshold of 10 $\mathrm{PeV}$.

It is a known feature that the farther the shower maximum is from the observation level, the greater is the radius of the Cherenkov ring. This is purely due to geometric effects of shower propagation. The propagation of the Cherenkov ring signature in the figure as the zenith angle increases is a manifestation of this. For an observation level of $2838 \mathrm{~m}$ above sea level, the average distance at which the Cherenkov ring falls is $\mathrm{d}_{\mathrm{Ch}} \approx 250 \mathrm{~m}$ for a shower of zenith angle $70^{\circ}$ and is $\mathrm{d}_{\mathrm{Ch}} \approx 150 \mathrm{~m}$ for a shower of zenith angle $60^{\circ}$. 


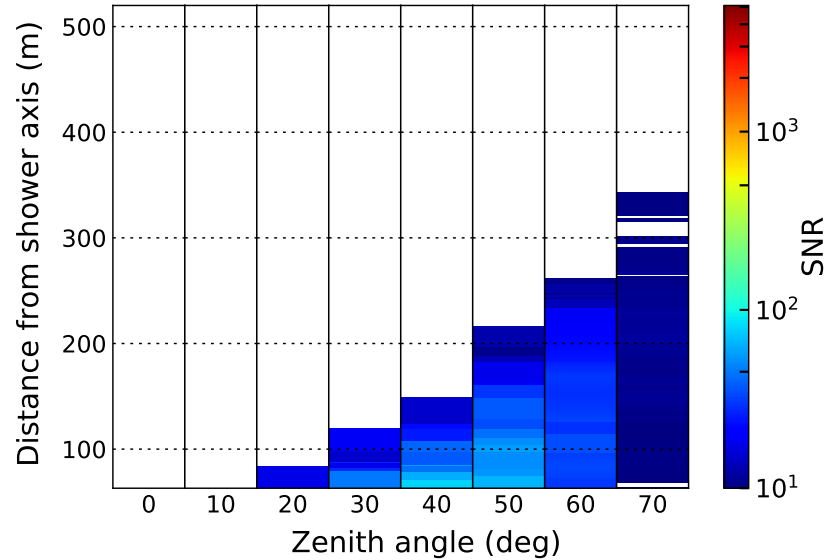

(a) $30-80 \mathrm{MHz}$

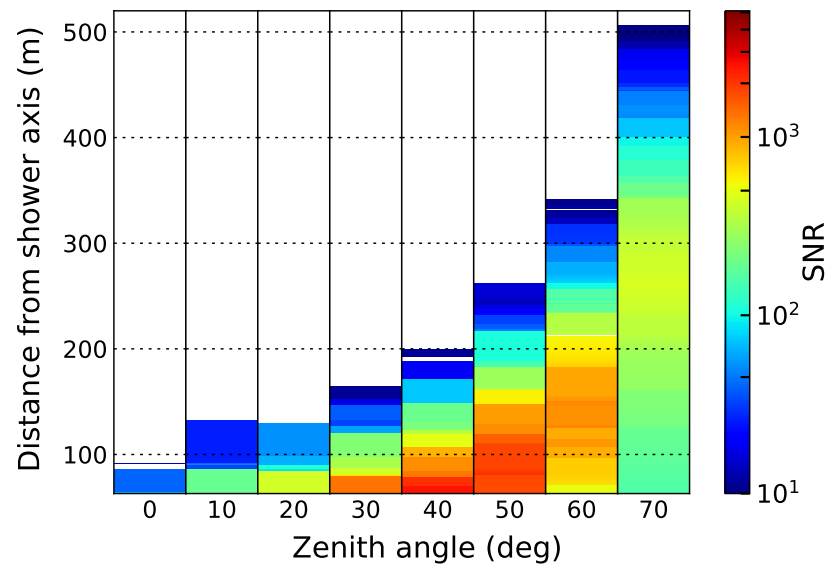

(b) $50-350 \mathrm{MHz}$

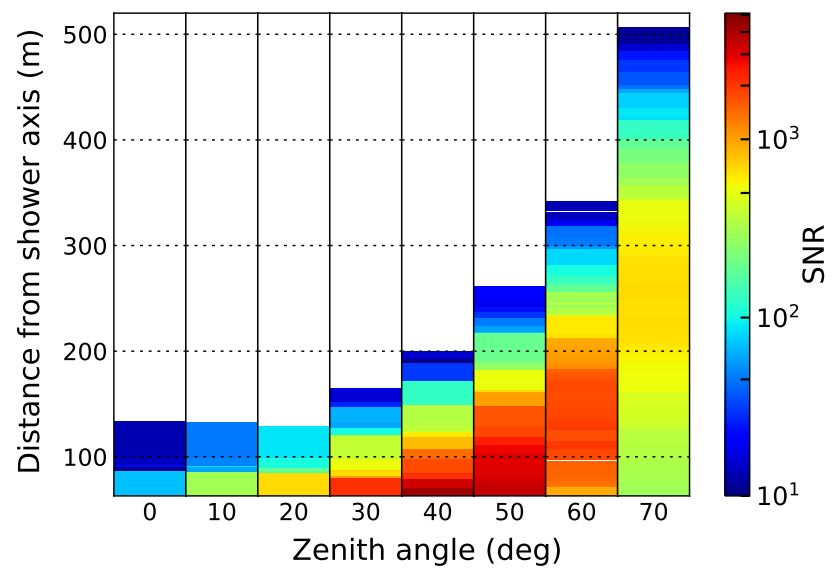

(c) $100-190 \mathrm{MHz}$

Fig. 6 Zenith angle dependence of the SNR for showers produced by $10 \mathrm{PeV}$ primary gamma ray with $\phi=0$. Each bin contains a typical shower for the respective zenith angle. At $\theta=70^{\circ}$ the shower illuminates almost the entire array $(\mathbf{b}, \mathbf{c})$

The total energy fluence of the radio signal at the ground increases up to the zenith angle where clipping effects are no longer observed. On an average, it was seen that for 10
PeV gamma-ray showers, the total radiated energy does not get clipped-off for zenith angles greater than $50^{\circ}$. For zenith angles greater than this, the total energy in the radio footprint remains nearly the same, but the area increases. This results in a lower power per unit area on the ground, causing a decrease in the SNR. The relatively lower signal-to-noise ratio for the $70^{\circ}$ shower in Fig. 6 as compared to the $60^{\circ}$ shower is an effect of this.

\subsection{Dependence on the azimuth angle}

Another parameter that the signal-to-noise ratio depends on is the azimuth angle of the shower. Variations in the azimuth angle result in changes in the geomagnetic angle. As a shower of zenith angle $61^{\circ}$ covers a range of azimuth angles from -180 to 180 degrees, the geomagnetic angle (at the South Pole, where the magnetic field is inclined to the vertical direction by $18^{\circ}$ ) varies from $43^{\circ}$ to $79^{\circ}$. This leads to an amplitude variation by a factor of $\frac{\sin \left(43^{\circ}\right)}{\sin \left(79^{\circ}\right)}=0.7$. We find that for gamma-ray showers with these range of orientations and with an energy of $10 \mathrm{PeV}$, the maximum value of the SNR varied with a standard deviation of $\sigma_{\mathrm{SNR}}=264$ with a mean value 1518. That is, with changing the azimuth angle there is a variation in the maximum value of the SNR by $17.4 \%$ about the mean. Apart from this, there is also a variation of the amplitude at a fixed azimuth angle due to shower-to-shower fluctuations which comes to $3.7 \%$ on an average. This will be further discussed in Sect. 4.3.

We can thus infer that for inclined air showers at the South Pole, there is not a strong variation of the signal-to-noise ratio as the azimuth angle varies. This is shown in Fig. 7. Here, gamma-ray showers each with an energy of $10 \mathrm{PeV}$ and an inclination of $61^{\circ}$ and with varying azimuth angles are shown. Thus it is justified to study the other effects only at one particular azimuth angle.

\subsection{Dependence on the primary energy}

The signal that is observed by the antennas will obviously depend on the energy of the primary particle. The SNR becomes weaker as the energy of the primary particle decreases. The signal-to-noise ratio of showers with gammaray and proton primaries with energies ranging from 1 to 9 $\mathrm{PeV}$, are shown in Fig. 8. These are showers with zenith angles of $61^{\circ}, 40^{\circ}$ and $70^{\circ}$, and are filtered to the band 100 $190 \mathrm{MHz}$.

If we use the optimal frequency band like $100-190 \mathrm{MHz}$, we will be able to lower the threshold of detection down to $1 \mathrm{PeV}$ for gamma-ray showers which have a zenith angle of $61^{\circ}$. For detection, it is required that a minimum of three antennas have a SNR above 10 . For $61^{\circ}$ showers, we can achieve this, provided we have at least three antennas within a distance of $\sim 50-180 \mathrm{~m}$ from the shower axis. This is mainly 


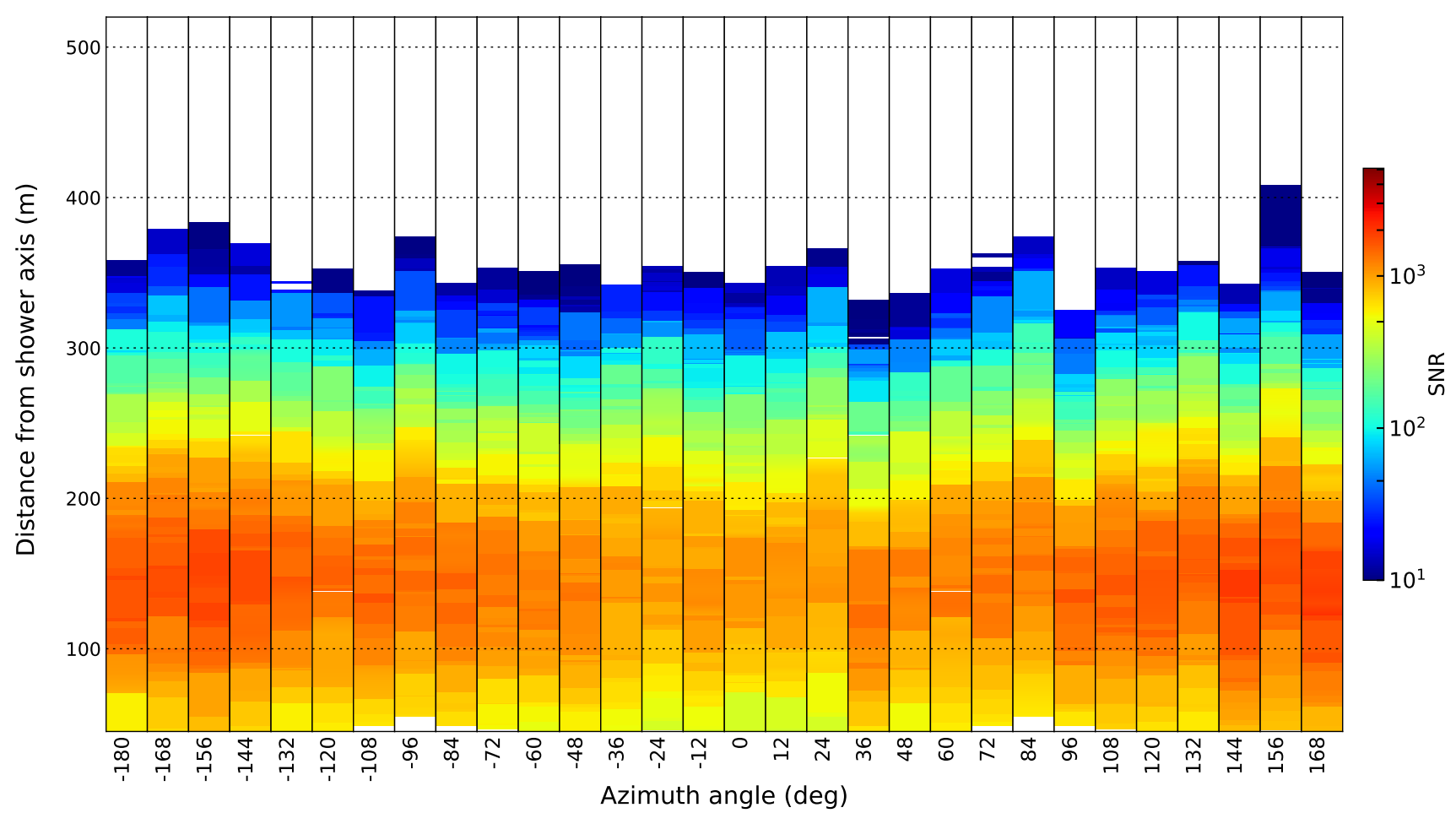

Fig. 7 Azimuth angle dependence of the SNR for $10 \mathrm{PeV}$ gamma-ray showers with zenith angle fixed to $61^{\circ}$. The figure shows a typical shower in each bin. The variation in the distance arises due to shower-to-shower fluctuations and azimuth angle variations

the area where the Cherenkov ring falls on the antenna array that gives a higher level of SNR. For proton showers of $61^{\circ}$ inclination, it is possible to lower the energy threshold to the level of $2 \mathrm{PeV}$ in the band 100-190 MHz.

In a similar manner, the energy threshold can be lowered for showers with zenith angles $40^{\circ}$ and $70^{\circ}$ as shown in Fig. 8 . For showers with $\theta=40^{\circ}$, we need at least three antennas within a distance of $\sim 80 \mathrm{~m}$ from the shower axis. This means that a much denser array is needed in this case. In the case of the $70^{\circ}$ showers, the minimum energy that can be detected is $2 \mathrm{PeV}$ and is nearly independent of how dense the array spacing is.

The showers shown in Fig. 8 are sample showers in these energy ranges. They will also have shower-to-shower fluctuations, because of which the amplitude detected in each antenna station will differ. Taking such fluctuations into account, gamma-ray induced air showers with zenith angles of $61^{\circ}$ and azimuth angles of $0^{\circ}$ were simulated with 11 simulations at each energy. Figure 9 shows the fluctuations in the maximum SNR and the maximum amplitude for these gamma-ray showers with energies ranging from 1 to $9 \mathrm{PeV}$. This is shown in the figure for a frequency range of 100-190 $\mathrm{MHz}$. These showers were seen to have an average relative standard deviation of the maximum SNR of $7.6 \%$ for all energies. Similarly, a $3.7 \%$ variation in the maximum amplitude is obtained.
It is seen that there is a clear correlation between the maximum SNR (or maximum amplitude) obtained and the energy of the primary particle. The maximum SNR was seen to be proportional to $E^{2}$ and the maximum amplitude $\propto E$. A fit of $\left.\mathrm{SNR}_{\max }=(17.04 \pm 0.43) \times E^{2.03 \pm 0.02}\right)$ was obtained. Similarly, the maximum amplitude was seen to be related to the energy as $\left.\mathrm{Amp}_{\max }=(8.04 \pm 0.10) \times E^{1.01 \pm 0.01}\right)$

Detection of air showers using the radio technique in the $\mathrm{PeV}$ energy range is something that has not been achieved so far. This study shows that such a detection is possible if the measurement is taken in the optimum frequency range, e.g. 100-190 MHz. This means that by using this frequency range, for radio air shower detectors, it is possible to search for gamma rays of PeV energy arriving at the South Pole, from the Galactic Center.

Such a method can indeed be used at other locations on the Earth, for increasing the probability of detection of lower energy air shower events. However, the Galactic Center may not be visible at all times. The exact threshold for detection may vary depending on the observation level, the magnetic field at these locations and the dimensions of the antenna array. The noise conditions of these areas will also affect the measurement. The use of the optimum frequencies will nevertheless increase the detection rate of inclined air showers and will lower the energy threshold. In addition, by using interferometric methods, the very conservative condition of SNR > 10 in 3 antennas can certainly be achieved. 


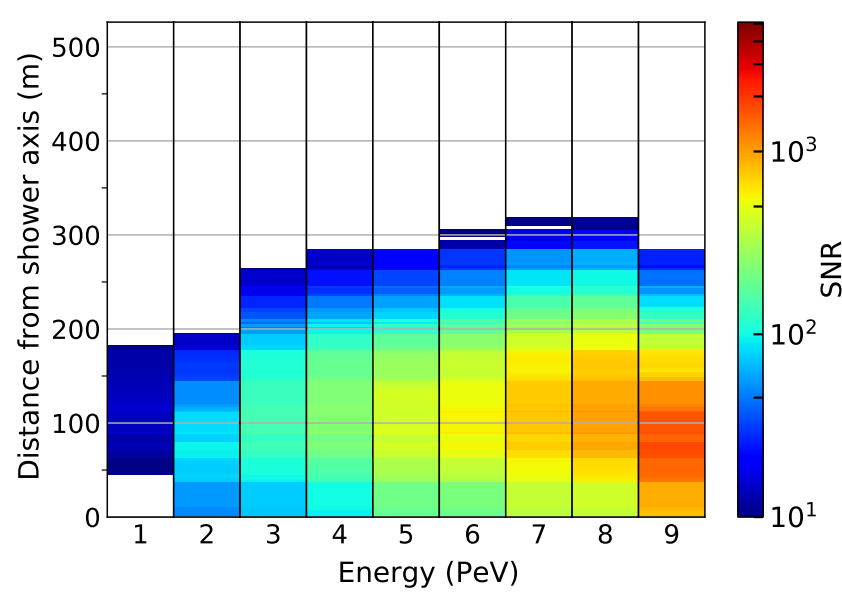

(a) $\gamma$-ray, $\theta=61^{\circ}, \phi=0^{\circ}\left(\alpha=79^{\circ}\right)$

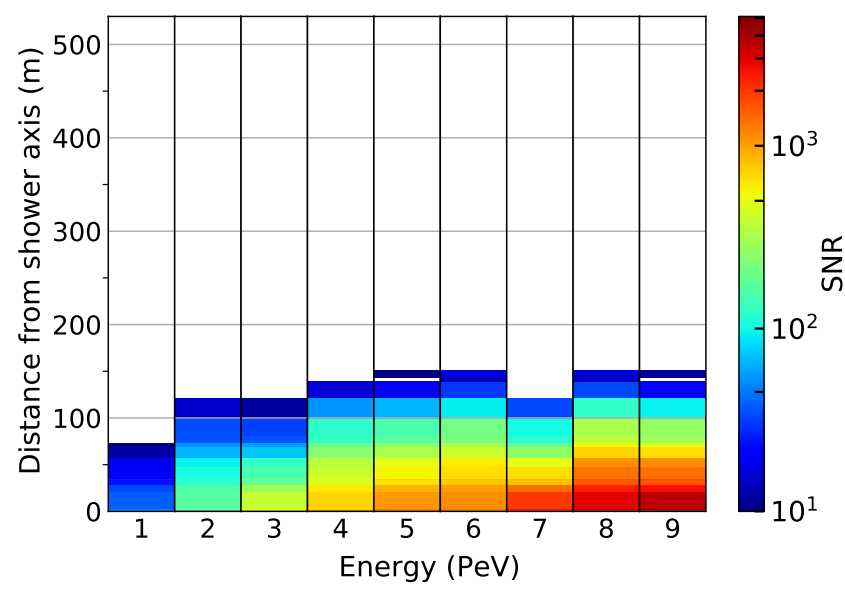

(b) $\gamma$-ray, $\theta=40^{\circ}, \phi=0^{\circ}\left(\alpha=58^{\circ}\right)$

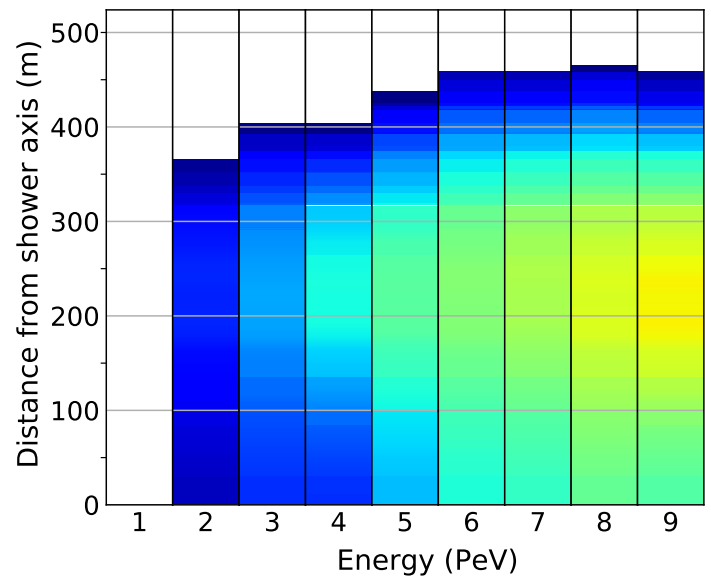

(c) $\gamma$-ray, $\theta=70^{\circ}, \phi=0^{\circ}\left(\alpha=88^{\circ}\right)$

Fig. 8 Left: gamma-ray induced showers at 100-190 MHz. Right: proton induced showers at $100-190 \mathrm{MHz}$. These are typical showers at these energies. Each bin contains one sample shower for the respective

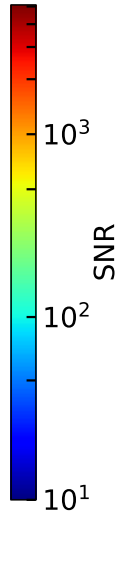

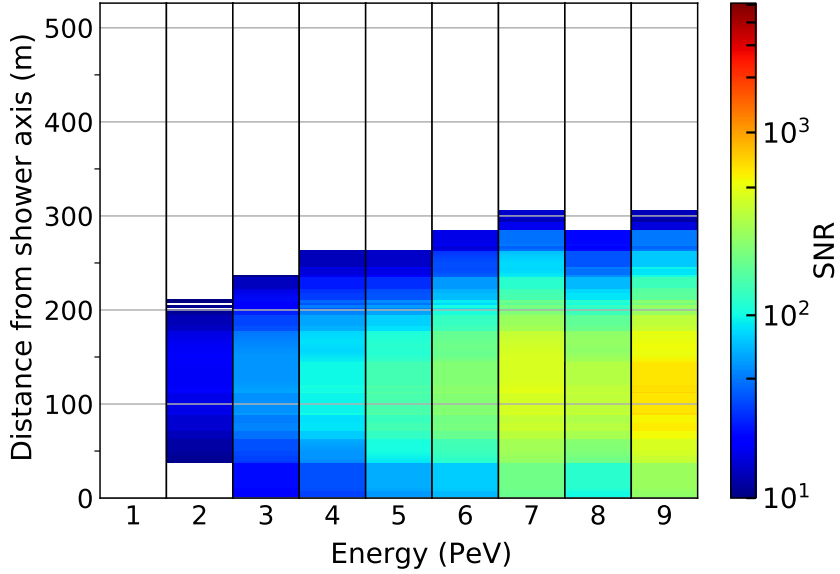

(d) proton, $\theta=61^{\circ}, \phi=0^{\circ}\left(\alpha=79^{\circ}\right)$

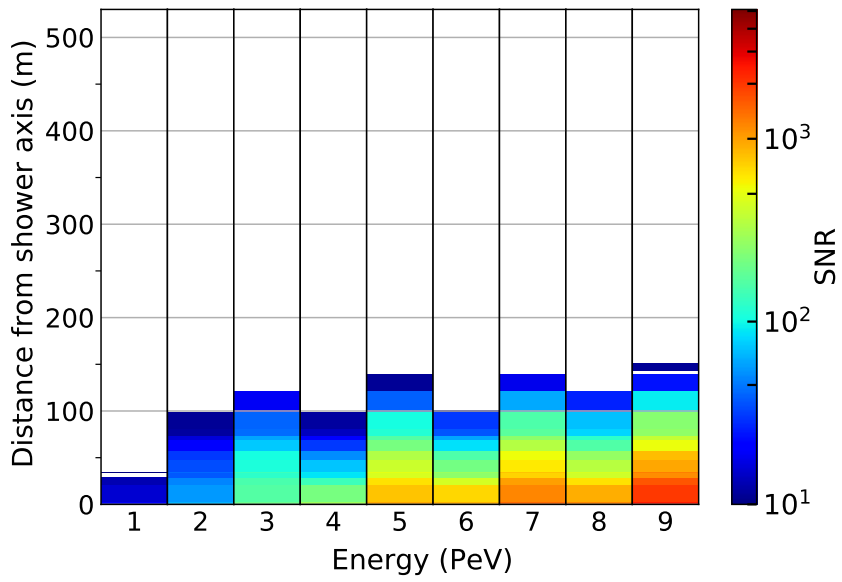

(e) proton, $\theta=40^{\circ}, \phi=0^{\circ}\left(\alpha=58^{\circ}\right)$

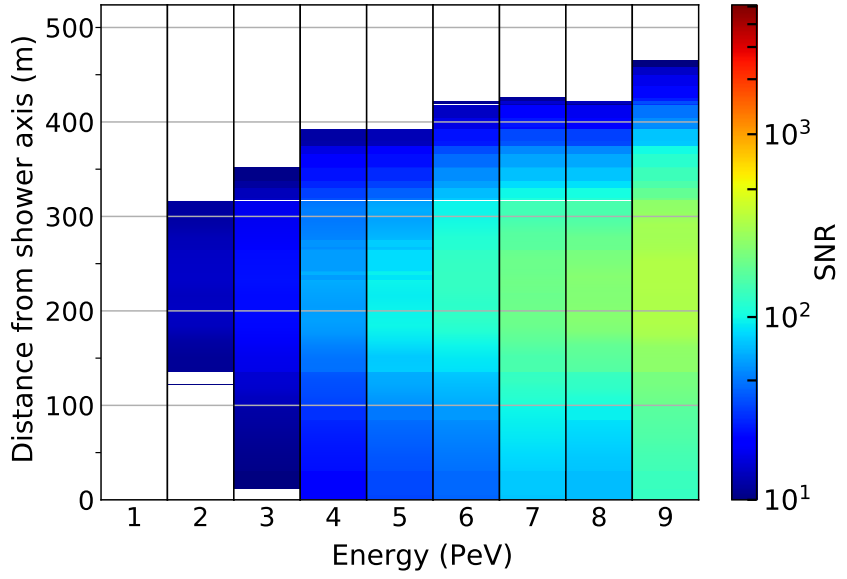

(f) proton, $\theta=70^{\circ}, \phi=0^{\circ}\left(\alpha=88^{\circ}\right)$

energy. The variation in the distances where antennas with SNR $>10$ are obtained for the same zenith angle arises due to shower-to-shower fluctuations 


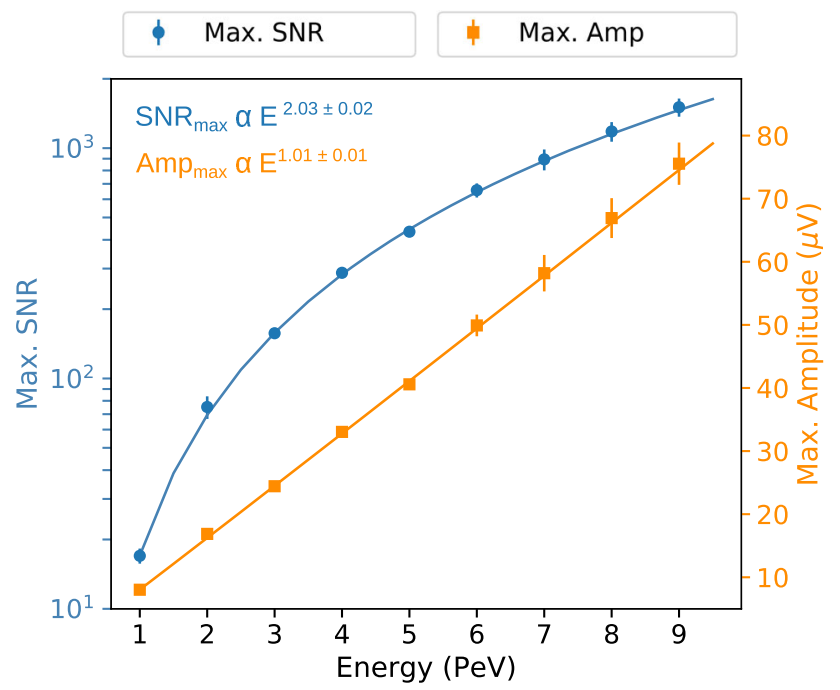

Fig. 9 The evolution of maximum SNR and maximum amplitude for gamma-ray showers with $\alpha=79^{\circ}$ and $\theta=61^{\circ}$ in the frequency band 100-190 MHz. The points represent the mean value and the standard deviation arising due to shower-to-shower fluctuations. The best fit to both set of simulated data points are shown, which has an $E^{2}$ nature for the maximum SNR and $E^{1}$ nature for the maximum amplitude

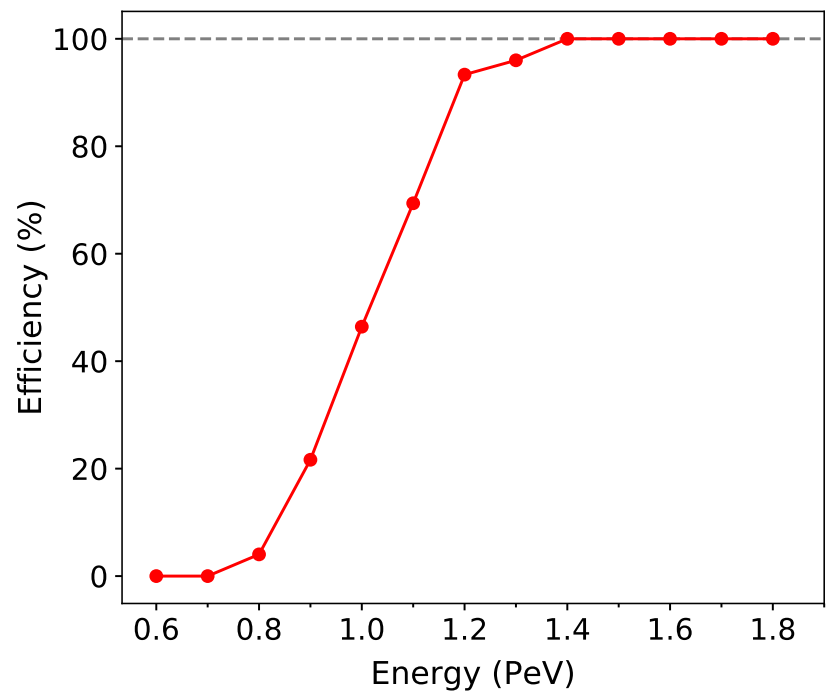

Fig. 10 Efficiency of detection at different energies. An SNR $>10$ in at least 3 antennas is applied as the condition for detection. This is tested for $100 \gamma$-ray showers with $\theta=61^{\circ}$ in each energy bin in the frequency band $100-190 \mathrm{MHz}$

\section{Efficiency of detection}

The results quoted in Sect. 4.3 will depend on fluctuations between different showers of $\mathrm{PeV}$ energy, that arrive at the antenna array with different azimuth angles and different core positions. These factors, along with shower-to-shower fluctuations, will affect the rate of detection of air showers produced by $\mathrm{PeV}$ gamma rays.
To have an estimate of this, 170 simulations of gamma-ray induced showers with an energy of $1 \mathrm{PeV}$ and zenith angle of $61^{\circ}$ were performed. These simulations had random azimuth angles and random core positions. Out of these showers, those with their core positions lying within a radius $\approx 564 \mathrm{~m}$ (corresponding to an area of $1 \mathrm{~km}^{2}$ ) from the center of the array were chosen. This reduced the sample size to 140 events. If more than three antennas in the array have SNR $>10$, the shower is detected. Upon conducting this test it was seen that these showers were detected with an efficiency of $47 \%$.

To have a better estimate of the energy where an efficiency of $100 \%$ is reached and the energy where the efficiency goes down to $0 \%$, simulations were done from 0.6 to $1.8 \mathrm{PeV}$ with 100 simulations in each energy bin, and with a bin width of $0.1 \mathrm{PeV}$. It was seen that an efficiency of $100 \%$ is reached for an energy of $1.4 \mathrm{PeV}$ and the efficiency goes down to $0 \%$ below $0.7 \mathrm{PeV}$. The efficiency curve for the simulated showers is shown in Fig. 10.

\section{Discussion}

As discussed in Sect. 4.3 it is possible to lower the energy threshold for radio detection by using an optimum frequency band. In the case of gamma-ray showers with a zenith angle of $61^{\circ}$, it is possible to lower the threshold down to $1 \mathrm{PeV}$ for the frequency band of $100-190 \mathrm{MHz}$, if we have an antenna array with an average spacing of $125 \mathrm{~m}$ at the South Pole. At other experimental locations, the threshold may vary depending on the specific environment of the region. This method can be used not only for the specific purpose of PeVatron detection, but also for improving our current understanding of air showers, e.g. the study of mass composition at energies starting from the $\mathrm{PeV}$ range.

The results presented here may vary depending on the exact noise that is present at the site of the experimental setup. On comparing with other available sky maps, the noise model by Cane predicts a level of noise that is slightly lower. For example, at a frequency of $110 \mathrm{MHz}$, the Cane model is seen to show around $15 \%$ less amplitude in the level of noise than that of other noise models like LFmap. This will introduce second order fluctuations in the SNR and has been neglected here. A more detailed study should also take these fluctuations into account. There will also be fluctuations depending on the local sidereal time. A thermal noise level of 300 $\mathrm{K}$ is considered in this study. Today, antennas with much lower system noise are available; e.g. the SKA-LOW prototype antenna, SKALA, has a system noise of about $40 \mathrm{~K}$ only [34]. The uncertainty arising from CoREAS can be estimated from the experimental tests made on CoREAS so far. Different air shower experiments determined CoREAS to be accurate on an absolute scale to better than $20 \%$ at frequencies up to $80 \mathrm{MHz}[35,36]$. This means that the uncertainty 
in the threshold due to the use of CoREAS is likely smaller than $20 \%$.

The detection potential of such an antenna array will also depend on the triggering capability. Triggers provided to the antennas by the IceTop array will not be fully efficient for $\mathrm{PeV}$ gamma-ray showers that are inclined with a zenith angle of $61^{\circ}$. Triggering is possible only if a particle from the air shower hits one of the IceTop tanks. The triggering capabilities of the future scintillator array is not yet studied in detail. Alternatively, if self-triggering of the antenna array is used, the energy threshold will rise depending on the broad-band radio interferences at the experimental site. The ARIANNA experiment has demonstrated that the conditions at Antarctica can be excellent for self-triggering [37].

A major challenge for the detection of these gamma rays is the background cosmic-ray flux which will be much larger than the gamma-ray flux. At energies above $0.8 \mathrm{PeV}$, a maximum of 8 gamma-ray events can be expected from the Galactic Center in one year, for a radio array with an area of $1 \mathrm{~km}^{2}$. Out of these, 5 events will be above $1.4 \mathrm{PeV}$, where we have a full efficiency of detection, assuming that events considered to be detectable will also be triggered. In order to distinguish these gamma-ray events, in a point-source scenario, from the background cosmic-ray events an angular resolution of $0.1^{\circ}$ or better, and a minimum gamma-hadron separation factor of 10 is required for a detection within a $5 \sigma$ confidence level in 3 years.

\section{Conclusions}

We have performed a simulation study for the detection of air showers produced by primary gamma rays of $\mathrm{PeV}$ energy. The focus is on showers of zenith angle $61^{\circ}$, since this is the direction from which $\mathrm{PeV}$ gamma rays will approach the IceCube Observatory from the Galactic Center. In order to find the best measurement parameters, CoREAS simulations have been done, assuming an antenna array at the positions of the IceTop stations.

The signal-to-noise ratio received at these antennas has mainly been focused on in this analysis. A scan of the possible frequency bands within which the experiment can operate shows that there is a range of frequencies within which the SNR is at the optimum level. One of these frequency bands, namely $100-190 \mathrm{MHz}$, has been used here for studying other shower dependencies. This is the first study that shows that moving to this frequency range will help in the detection of inclined air showers. It will even help in lowering the energy threshold for gamma-ray showers with a zenith angle of $61^{\circ}$ down to $\approx 1 \mathrm{PeV}$ at the South Pole.

For a hybrid array of $1 \mathrm{~km}^{2}$ area and an average antenna spacing of $125 \mathrm{~m}$, with an operating frequency band of 100 $190 \mathrm{MHz}, 61^{\circ}$ gamma-ray showers of $1 \mathrm{PeV}$ can be detected with an efficiency of $47 \%$. This number was determined for showers whose cores fall within a circular region around the array center covering an area of $1 \mathrm{~km}^{2}$. An antenna array with an average spacing of $125 \mathrm{~m}$ has been used in this case. We can reach a full efficiency above $1.4 \mathrm{PeV}$, and have a non-zero rate of detection above $0.8 \mathrm{PeV}$. Due to the simplifications in the simulation studies, the experimentally achievable thresholds may vary by a few tens of percents. They could even be lowered further with the usage of sophisticated hardware or by using interferometric detection techniques. Using even the simple radio setup considered in this paper at the IceCube location will give us a chance for detecting $\mathrm{PeV}$ gamma rays from the Galactic Center.

Acknowledgements We would like to acknowledge the support of the IceCube collaboration and the members of the IceCube-Gen2 group at KIT and DESY-Zeuthen. We would also like to thank Anna Nelles and Larissa Paul for their very useful inputs.

Open Access This article is distributed under the terms of the Creative Commons Attribution 4.0 International License (http://creativecomm ons.org/licenses/by/4.0/), which permits unrestricted use, distribution, and reproduction in any medium, provided you give appropriate credit to the original author(s) and the source, provide a link to the Creative Commons license, and indicate if changes were made. Funded by $\mathrm{SCOAP}^{3}$.

\section{Appendix A: Estimation of the number of gamma-ray events with $\mathrm{PeV}$ energies}

It is essential to have an estimate of the number of gammaray events of $\mathrm{PeV}$ energy expected to approach the detector from the direction of the Galactic Center. For this, we have to extrapolate the flux of $\mathrm{TeV}$ gammas that has been observed by H.E.S.S.. A simple extrapolation to $\mathrm{PeV}$ energies, without any cut-off (which is preferred by the H.E.S.S. data points), is shown in Fig. 11. A spectrum with cut-off at energies like 1,10 or $100 \mathrm{PeV}$ is also possible. Here we mainly consider the best-case scenario, that is a spectrum without any cut-off. A spectrum of $\frac{d N}{d E} \propto E^{-2.32}$ is used for the extrapolation, which is the best fit to the H.E.S.S. data points.

The extrapolated flux will also get attenuated due to the CMB [38]. This leads to a survival probability of the gamma rays from the extrapolated flux given by $\approx \frac{1}{e^{L_{\text {dis }} / L_{\text {atten }}}}$, where $L_{\mathrm{dis}}$ is the distance traveled by the gamma rays (here it is the distance between the Earth and the Galactic Center $\approx 8.5$ $\mathrm{kpc})$ and $L_{\text {atten }}$ is the attenuation lengths of the gamma rays at different energies. The resulting spectrum after attenuation is also shown in Fig. 11. Finally, the efficiency of detection of the radio array at various energies is also taken into consideration. The resulting flux that will be seen by the antenna array is shown by the black curve in the figure. From this method of extrapolation, an estimate of the expected number of events above $\mathrm{PeV}$ energies, detected in a year in a array of area $1 \mathrm{~km}^{2}$, has been derived. Since the Galactic Center lies at an inclination of $61^{\circ}$ at the South Pole, the area of cover- 


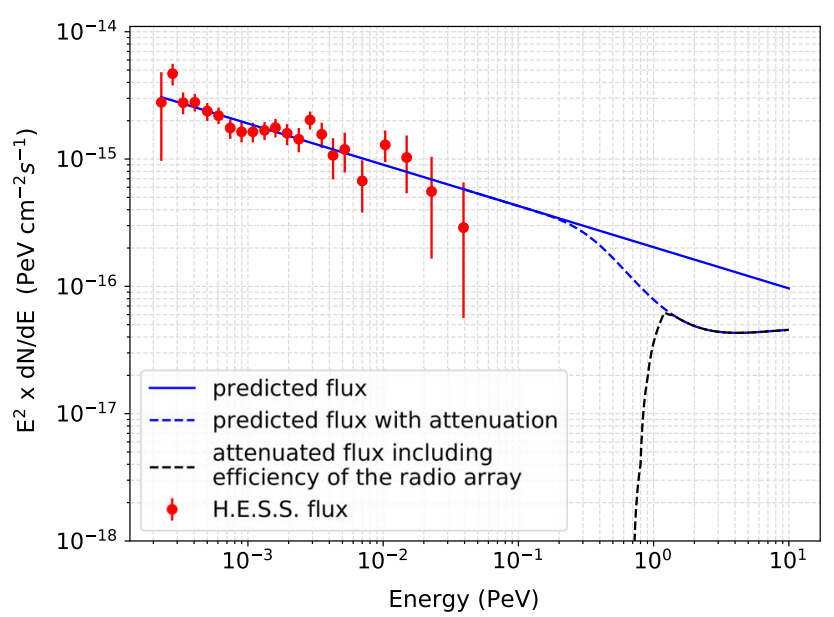

Fig. 11 Flux as seen by H.E.S.S. [3], with a simple $E^{-2.32}$ extrapolation. The gamma-ray flux will get attenuated due to interactions with the CMB. The detectable flux takes the detection efficiency of the radio array into account

age of the array has to be weighted by a geometry factor of cosine $\left(61^{\circ}\right)$.

The expected number of events above $0.8 \mathrm{PeV}$, where we have a non-zero efficiency of detection, and above $1.4 \mathrm{PeV}$, where we have a full efficiency, are shown in Table 1. Here, we have made the assumption that the events that can be detected by the radio array will also be triggered. The number of events before and after folding through the detection efficiency are shown in the table. These numbers are evaluated using the attenuated flux of gamma rays.

To obtain an estimate of the required gamma-hadron separation factor, we compared the expected number of gamma rays in 3 years with the number of cosmic rays that IceTop can see in 3 years within a region of the sky with a diameter of $0.1^{\circ}$. Above $0.8 \mathrm{PeV}$, the expected number of cosmic rays is $\approx 289.3$ and gamma rays is $\approx 23.8$. This means that for a detection within a confidence level of $5 \sigma$, we will need a separation factor of $\approx 12.7$ On the other hand, if we consider the number of events above $1.4 \mathrm{PeV}$ (gamma rays $\approx 15.4$ and cosmic rays $\approx 99.5$ ), we require a separation factor of $\approx 10.5$. The gamma-hadron separation can be done by using the information of the shower maximum or by using the different muon content of showers from these primaries. The optimization of this requires a separate, deeper study.

\section{Appendix B: Generating a noise trace}

A model for the Galactic noise developed by Cane [30] is used for the following discussion. The Galactic noise is provided in units of brightness $(B(v))$ and is expressed as a function of frequency. Assuming the source of Galactic noise to be a blackbody and hence using the Rayleigh-Jeans law, we can relate the brightness to its brightness temperature.
$B(v)=2 k_{\mathrm{B}} T \frac{v^{2}}{c^{2}} \quad\left[\mathrm{Wm}^{-2} \mathrm{sr}^{-1} \mathrm{~Hz}^{-1}\right]$

where $\mathrm{k}_{\mathrm{B}}$ is the Boltzmann's constant and T is the brightness temperature.

We can add thermal noise due to the electronics of the receiving system to the brightness temperature in order to obtain the total noise temperature.

$T_{\text {tot }}=T_{\text {brightness }}+T_{\text {thermal }}$

The electromagnetic power of the noise obtained in the frequency band $\delta \nu$ from solid angle $\mathrm{d} \Omega$ by an antenna of effective area of $A_{\text {eff }}$ is,

$P_{\nu}(\theta, \phi)=\frac{1}{2} B(v) \mathrm{d} \Omega A_{\text {eff }}(\theta, \phi) \delta v$

Here, a factor of $1 / 2$ has to be added in order to account for the fact that the antenna can extract power only from one of the polarizations of the incoming electromagnetic wave.

The Poynting flux per unit frequency is obtained by integrating the brightness over the solid angle.

$$
\begin{aligned}
S & =\int B(v) \mathrm{d} \Omega \quad\left[\mathrm{Wm}^{-2} \mathrm{~Hz}^{-1}\right] \\
& =\frac{2 k_{\mathrm{B}} v^{2}}{c^{2}} \int T(\theta, \phi) \mathrm{d} \Omega
\end{aligned}
$$

The Poynting flux within the frequency interval of $\delta v$ is then,

$$
\begin{aligned}
S_{v} & =S \delta v \quad\left[\mathrm{Wm}^{-2}\right] \\
& =\frac{2 k_{\mathrm{B}} v^{2} \delta v}{c^{2}} \int T(\theta, \phi) \mathrm{d} \Omega
\end{aligned}
$$

Again, the Poynting flux extracted at the antenna is $S_{\text {rec }}=\frac{S_{v}}{2}$ for reasons of polarization matching.

We can relate the Poynting flux to the electric field delivered to the antenna as,

$|\vec{S}|=\frac{1}{2 n Z_{0}}|\vec{E}|^{2}$

where $Z_{0}=376.7303 \mathrm{Ohm}$ is the vacuum impedance.

Taking the refractive index of air to be 1 , the amplitude of the electric field at the antenna because of the Galactic noise can then be obtained from the Poynting flux as,

$$
\begin{aligned}
|\vec{E}| & =\sqrt{S_{\mathrm{rec} 2 Z_{0}}} \quad[\mathrm{~V} / \mathrm{m}] \\
& =\sqrt{\frac{1}{2} 2 Z_{0} \frac{2 k_{\mathrm{B}} v^{2} \delta v}{c^{2}}} \int T(\theta, \phi) \mathrm{d} \Omega
\end{aligned}
$$

Thus, the voltage developed at the antenna is

$$
V(v)=\vec{E} \cdot \overrightarrow{l_{\mathrm{eff}}} \quad[\mathrm{V}]
$$


Table 1 Estimated number of events per year obtained from the extrapolation of the attenuated gamma-ray flux, with and without detector efficiency limits

\begin{tabular}{lll}
\hline & $\begin{array}{c}N_{\text {events }}\left(>E_{0}\right) \\
(1 \text { year })\end{array}$ & $\begin{array}{c}N_{\text {events }}\left(>E_{0}\right) \\
\times \text { efficiency }\end{array}$ \\
\hline $\begin{array}{c}E_{0}=0.8 \mathrm{PeV} \\
(\text { efficiency }>0)\end{array}$ & 11.5 & 7.9 \\
$\begin{array}{c}E_{0}=1.4 \mathrm{PeV} \\
\text { (full efficiency) }\end{array}$ & 5.1 & 5.1 \\
\hline
\end{tabular}

Since we have already taken into account that the polarization should match, we can multiply the modulus of the field and the modulus of the antenna height to obtain the voltage. Of course, this simplification cannot be done for a noise model with directional dependence. The received voltage is now given by

$V(v)=\sqrt{2 Z_{0} \frac{k_{\mathrm{B}} v^{2} \delta v}{c^{2}}} \int T(\theta, \phi) l_{\mathrm{eff}}(\theta, \phi) \mathrm{d} \Omega$

Since the model used has the temperature to be independent of $\theta$ and $\phi, T(\theta, \phi)=T$ can be taken out of the integral.

The amplitude extracted from the model has no phase information of the incoming noise. We can add random phases to the amplitude since noise indeed behaves randomly.

$V(v)=V(\nu) * \exp (-i \varphi)$

$\varphi$ is a random number that is generated between 0 and $2 \pi$.

Finally, we can convert the amplitude to the time domain using Inverse Fourier Transform:

$V(v) \longrightarrow V(t)$.

Of course, this is only an average behavior of the noise. One can also assume variations in the extracted amplitude about this average noise.

\section{References}

1. T. Huege, Radio detection of cosmic ray air showers in the digital era. Phys. Rep. 620, 1-52 (2016). https://doi.org/10.1016/j. physrep.2016.02.001

2. Frank G. Schröder, Radio detection of cosmic-ray air showers and high-energy neutrinos. Prog. Part. Nucl. Phys. 93, 1-68 (2017). https://doi.org/10.1016/j.ppnp.2016.12.002

3. A. Abramowski et al., (HESS Collaboration), Acceleration of petaelectronvolt protons in the Galactic Centre. Nature 531, 476 (2016). https://doi.org/10.1038/nature17147

4. M.C. Gonzalez-Garcia, Francis Halzen, Soumya Mohapatra, Identifying Galactic PeVatrons with neutrinos. Astropart. Phys. 31, 437-444 (2009). https://doi.org/10.1016/j.astropartphys.2009.05. 002
5. F. Halzen, A. Kappes, A.O. Murchadha, Gamma-ray Astronomy with Muons: Sensitivity of IceCube to PeVatrons in the Southern Sky. Phys. Rev. D 80, 083009 (2009). https://doi.org/10.1103/ PhysRevD.80.083009

6. A. Achterberg et al., (IceCube Collaboration), First year performance of the IceCube Neutrino Telescope. Astropart. Phys. 26, 155-173 (2006). https://doi.org/10.1016/j.astropartphys.2006.06. 007

7. R. Abbasi et al., (IceCube Collaboration), IceTop: the surface component of IceCube. Nucl. Instrum. Methods A700, 188-220 (2013). https://doi.org/10.1016/j.nima.2012.10.067

8. S. Kunwar, T. Huber, J. Kelley, D. Tosi, for the IceCube-Gen2 collaboration, The IceTop Scintillator Upgrade. In Proceedings, 35th International Cosmic Ray Conference, PoS(ICRC 2017)401 (2017)

9. J. van Santen, for the IceCube-Gen2 collaboration, IceCube-Gen2: the next-generation neutrino observatory for the South Pole. In Proceedings, 35th International Cosmic Ray Conference, PoS(ICRC 2017)991 (2017)

10. S. Euler, J. Gonzalez, B. Roberts, for the IceCube-Gen2 collaboration, Simulation studies for a surface veto array to identify astrophysical neutrinos at the South Pole. In 34th International Cosmic Ray Conference, PoS(ICRC2015)1070 (2015)

11. D. Seckel, Cosmic ray science potential for an extended surface array at the IceCube observatory. In Proceedings, 34th International Cosmic Ray Conference: The Hague, The Netherlands, July 30-August 6 2015, PoS(ICRC 2015)694 (2015)

12. J. Auffenberg, for the IceCube-Gen2 collaboration, IceAct: Imaging Air Cherenkov Telescopes with SiPMs at the South Pole for IceCube-Gen2. In Proceedings, 35th International Cosmic Ray Conference, PoS(ICRC 2017)1055 (2017)

13. Sebastian Boser, A Radio Air-Shower Test Array (RASTA) for IceCube. Nucl. Instrum. Methods A662, S66-S68 (2012). https:// doi.org/10.1016/j.nima.2010.11.164

14. P. Allison et al., Performance of two Askaryan Radio Array stations and first results in the search for ultrahigh energy neutrinos. Phys. Rev. D 93(8), 082003 (2016). https://doi.org/10.1103/PhysRevD. 93.082003

15. F.D. Kahn, I. Lerche, Radiation from cosmic ray air showers. Proc. R. Soc. Lond. A Math. Phys. Eng. Sci. 289(1417), 206-213 (1966). ISSN 0080-4630. https://doi.org/10.1098/rspa.1966.0007. http:// rspa.royalsocietypublishing.org/content/289/1417/206

16. O. Scholten, K. Werner, F. Rusydi, A macroscopic description of coherent geo-magnetic radiation from cosmic ray air showers. Astropart. Phys. 29, 94-103 (2008). https://doi.org/10.1016/j. astropartphys.2007.11.012

17. G.A. Askaryan, Excess negative charge of an electron-photon shower and the coherent radio emission from it. Soviet Phys. JETP 14, 441 (1962)

18. G.A. Askaryan, Coherent radio emission from cosmic showers in air and in dense media. Soviet Phys. JETP 21, 658 (1965)

19. K.D. de Vries, A.M. van den Berg, O. Scholten, K. Werner, Coherent Cherenkov radiation from cosmic-ray-induced air showers. Phys. Rev. Lett. 107, 061101 (2011). https://doi.org/10.1103/ PhysRevLett.107.061101

20. M.G. Labate, P. Dewdney, R. Braun, M. Waterson, J. Wagg, The SKA low-frequency telescope: performance parameters and constraints on the array configuration. In 2017 11th European Conference on Antennas and Propagation (EUCAP), pp. 2259-2263 (2017). https://doi.org/10.23919/EuCAP.2017.7928622

21. P. Abreu et al., (Pierre Auger Collaboration), Antennas for the detection of radio emission pulses from cosmic-ray induced air showers at the Pierre Auger Observatory. J. Instrum. 7(10), P10011 (2012). http://stacks.iop.org/1748-0221/7/i=10/a=P10011

22. P.A. Bezyazeekov et al., (LOFAR Collaboration), Measurement of cosmic-ray air showers with the Tunka Radio Extension (Tunka- 
Rex). Nucl. Instrum. Methods A802, 89-96 (2015). https://doi.org/ 10.1016/j.nima.2015.08.061

23. P. Schellart et al., (LOFAR Collaboration), Detecting cosmic rays with the LOFAR radio telescope. Astron. Astrophys. 560, A98 (2013). https://doi.org/10.1051/0004-6361/201322683

24. Olga Kambeitz, Measurement of horizontal air showers with the Auger Engineering Radio Array. EPJ Web Conf. 135, 01015 (2017). https://doi.org/10.1051/epjconf/201713501015

25. T. Huege, A. Haungs, Radio detection of cosmic rays: present and future. JPS Conf. Proc. 9, 010018 (2016). https://doi.org/10.7566/ JPSCP.9.010018

26. T. Huege, M. Ludwig, C.W. James, Simulating radio emission from air showers with CoREAS. AIP Conf. Proc. 1535, 128 (2013). https://doi.org/10.1063/1.4807534

27. D. Heck, G. Schatz, T. Thouw, J. Knapp, J.N. Capdevielle, CORSIKA: a Monte Carlo code to simulate extensive air showers. FZKA-6019 (1998)

28. E.-J. Ahn, R. Engel, T.K. Gaisser, P. Lipari, T. Stanev, Cosmic ray interaction event generator sibyll 2.1. Phys. Rev. D 80, 094003 (2009). https://doi.org/10.1103/PhysRevD.80.094003

29. Timothy C.A. Molteno, NEC2++: an NEC-2 compatible Numerical Electromagnetics Code. Technical Report ISSN 1172-496X, Electronics Technical Reports No. 2014-3 (2014)

30. H.V. Cane, Spectra of the non-thermal radio radiation from the galactic polar regions. MNRAS 189, 465-478 (1979)

31. M. Duvernois, The Radio Air Shower Test Array (RASTA) enhancing the IceCube observatory. In Proceedings, 32nd International Cosmic Ray Conference (ICRC 2011), vol. 3, p. 251. https://doi.org/10.7529/ICRC2011/V03/1102. https://inspirehep. net/record/1352342/files/v3_1102.pdf
32. P. Allison et al., (ARA Collaboration), Design and initial performance of the Askaryan Radio Array Prototype EeV Neutrino Detector at the South Pole. Astropart. Phys. 35, 457-477 (2012). https://doi.org/10.1016/j.astropartphys.2011.11.010

33. Alexander Aab et al., (Pierre Auger Collaboration), Energy estimation of cosmic rays with the engineering radio array of the Pierre Auger Observatory. Phys. Rev. D 93(12), 122005 (2016). https:// doi.org/10.1103/PhysRevD.93.122005

34. E. de Lera Acedo, N. Razavi-Ghods, N. Troop, N. Drought, A.J. Faulkner, A.J. Faulkner, SKALA, a log-periodic array antenna for the SKA-low instrument: design, simulations, tests and system considerations. Exp. Astron. 39, 567-594 (2015). https://doi.org/10. 1007/s10686-015-9439-0

35. W.D. Apel et al., (LOPES Collaboration), Improved absolute calibration of LOPES measurements and its impact on the comparison with REAS 3.11 and CoREAS simulations. Astropart. Phys. 75, 72-74 (2016a). https://doi.org/10.1016/j.astropartphys.2015. 09.002

36. W.D. Apel et al., A comparison of the cosmic-ray energy scales of Tunka-133 and KASCADE-Grande via their radio extensions Tunka-Rex and LOPES. Phys. Lett. B 763, 179-185 (2016b). https://doi.org/10.1016/j.physletb.2016.10.031

37. S.W. Barwick et al., Radio detection of air showers with the ARIANNA experiment on the Ross Ice Shelf. Astropart. Phys. 90, 50 68 (2017). https://doi.org/10.1016/j.astropartphys.2017.02.003

38. Silvia Vernetto, Paolo Lipari, Absorption of very high energy gamma rays in the Milky Way. Phys. Rev. D 94(6), 063009 (2016). https://doi.org/10.1103/PhysRevD.94.063009 